\title{
Las "licenciaturas" como grado académico: Una revisión bibliográfica en el contexto de Costa Rica'
}

\author{
The "Licentiate" As an academic degree: A Literature Review in the Costa Rica Context"
}

\section{As "licenciaturas" como um grau académico: Uma revisão da literatura no contexto da Costa Rica}

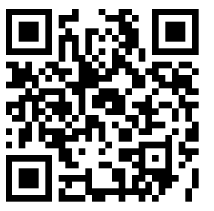

Heredia, Costa Rica
flizano@una.cr
http://orcid.org/0000-0001-8789-2985
Carmen Cordero-Esquivel
Universidad Nacional
Escuela de Informática
Heredia, Costa Rica
ccordero@una.cr
(D) http://orcid.org/0000-0003-4018-1275

Recibido 1 de marzo de 2016 • Corregido 31 de mayo de 2017 • Aceptado 19 de julio de 2017

Received 1 de marzo de 2016 • Revised 31 de mayo de 2017 • Accepted 19 de julio de 2017

Recebido 1 de marzo de 2016 • Revisado 31 de mayo de 2017 • Aprovado 19 de julio de 2017

\footnotetext{
'Este artículo se escribió en el contexto del proyecto de investigación 0001-14 denominado "Estudio de factibilidad y propuesta de creación de nuevos programas a nivel de posgrado en computación para la Escuela de Informática" (Lizano y Cordero, 2015), el cual se desarrolló del 1 de julio del 2014 al 31 de diciembre del 2015.

${ }^{2}$ This paper was written in the context of the research project 0001-14 entitled "Feasibility study and proposal for the creation of new postgraduate programs in computer science for the School of Computing Science" (Lizano y Cordero, 2015), a study developed from July 1, 2014 to December 31, 2015.

${ }^{3}$ Este artigo foi escrito no contexto do projeto de pesquisa 0001-14 intitulado "Estudo de viabilidade e proposta para a criação de novos programas de pós-graduação em ciências da computação para a Escola de Informática" (Lizano y Cordero, 2015), estudo desenvolvido entre 1 de Julho de 2014 e 31 de Dezembro de 2015.

${ }^{4}$ Bachiller Ingeniería Informática (ULACIT, San José, Costa Rica), Máster en Ingeniería de Sistemas (ITCR, Cartago, Costa Rica), PhD Ciencias de la Computación (Aalborg University, Aalborg, Dinamarca). Certified Tester (Software Testing - CTFL-ISTQB). Su desempeño profesional ha sido en programación, análisis de sistemas, aseguramiento de calidad del software, consultoría, auditoria de sistemas. Trabaja como profesor a tiempo completo en la Universidad Nacional, Heredia, Costa Rica. Su área de interés de investigación se centra en las evaluaciones de usabilidad y cómo integrarlas en el proceso de desarrollo de software, aseguramiento de la calidad y testing remoto de usabilidad.

${ }^{5}$ Bachiller en Enseñanza de Computación e Informática (Universidad Nacional de Costa Rica); Máster en Administración de Tecnología de la Información, Énfasis en Administración de la Información (Universidad Nacional de Costa Rica). Se ha desempeñado como docente universitaria, asesora en informática, coordinadora académica de un postgrado en tecnología e informática educativa y Coordinadora del Programa de Posgrado de la Escuela de Informática. Tiene experiencia en la incorporación de tecnologías digitales para favorecer los procesos de enseñanza y el aprendizaje, software educativo y entornos de aprendizaje (LMS). así como el diseño de planes de estudio. El área de interés de investigación es la interacción persona-computador.
} 
doi: http://dx.doi.org/10.15359/ree.21-3.4

URL: http://www.una.ac.cr/educare

CORREO: educare@una.cr

Resumen: Este artículo tiene el objetivo de presentar una revisión bibliográfica efectuada para verificar el uso actual del grado académico de "licenciatura". En el alcance de estudio existe una discusión respecto a la pertinencia de la continuidad del ofrecimiento de este grado en temáticas relacionadas con la computación. Esta discusión cobra especial relevancia en el contexto de proceso del planteamiento de nuevos programas de estudio de grado y posgrado. Los estudios considerados en este documento abarcan el periodo comprendido entre 1981 y el presente. Los criterios para analizar la información bibliográfica se enfocan de lo general a lo especifico, iniciando por una revisión del estado de la situación global para luego pasar al contexto de Costa Rica. Adicionalmente, esta revisión tiene una orientación basada en prácticas o aplicaciones, un objetivo de identificación de ideas centrales, una perspectiva de exposición de una posición, una cobertura representativa, una organización histórica-conceptual y está dirigida a una audiencia general y particularmente a una enfocada en generación de políticas. Los resultados muestran una inexistencia del grado de licenciatura en computación en regiones más desarrolladas como USA y Europa, y una decadencia latinoamericana y nacional respecto de la relevancia de estas.

Palabras claves: Computación; planes de estudio; licenciatura; informática.

Abstract: This paper aims to present a literature review made in order to verify the current use of the "licentiate" academic degree. In the scope of this study, there is a debate about the pertinence of continuing to offer such academic degree on topics related to computing. This debate has special relevance in the context of the process of proposing new graduate and postgraduate programs. The literature included in this paper was published from 1981 to the present. The criteria used to analyze bibliographic information focus on aspects going from the general to the specific level, starting with a review of the global situation to continue later to approach the specific context of Costa Rica. In addition, this literature review has an orientation based on the following aspects: practices or applications, an objective to identify main ideas, a perspective to display a position, a representative coverage, and a historical-conceptual organization; and this orientation is targeting a general audience, and specially policymakers. Results shows no presence of the licentiate degree in computing in developed regions like USA and Europe, and a decadence in the relevance of such degrees in Latin America and Costa Rica.

Keywords: Computer Sciences; study plans; licentiate degree; Computing science.

Resumo: O objetivo desse artigo é apresentar uma revisão da literatura existente para verificar o uso atual do grau académico de "Licenciatura". No âmbito de estudo existe um debate sobre a relevância da continuidade em oferecer esse grau em matérias relacionadas com computação. Esta discussão é particularmente importante, contextualizada no processo de planejar novos currículos de graduação e pós-graduação. Os estudos aqui considerados compreendem desde o período de 1981 até a atualidade. Os critérios para análise da informação bibliográfica enfocaram o tema desde o aspecto geral até o específico, começando com uma revisão do estado da situação global e, em seguida, passar para o contexto da Costa Rica. Além disso, a revisão está orientada e baseada em práticas ou aplicações, como objetivo, identificar as ideias principais, uma perspectivas de expor uma posição, uma cobertura representativa, uma organização histórico-conceitual e é voltada para o público em geral e, particularmente, focada em gerar políticas. Os resultados mostram a ausência do grau de licenciatura em computação nas regiões mais desenvolvidas, como EUA e Europa, e um declínio relacionado à importância desse grau na América Latina e na Costa Rica.

Palavras-chave: Computação; currículos; Licenciatura; informática. 


\section{Introducción}

Este artículo fue desarrollado dentro del contexto del proyecto de investigación (0001-14), llamado“Estudio de factibilidad y propuesta de creación de nuevos programas a nivel de posgrado en computación para la Escuela de informática" (Lizano y Cordero, 2015). El objetivo general de este proyecto es desarrollar un estudio de factibilidad y escribir un documento base de discusión para la creación de nuevos programas de posgrado en el área de computación o áreas afines. En el primer objetivo específico del proyecto, se expone que se deberá diagnosticar la situación actual y perspectivas de los actuales programas de Licenciatura de la Escuela de Informática. Como indicador de logro del citado objetivo específico se debe desarrollar un documento diagnóstico de la situación actual del grado de licenciatura ofrecido por la Escuela de Informática.

Este artículo tiene el objetivo de presentar los resultados de una revisión bibliográfica sistemática hecha en el contexto del estado de la situación del grado de licenciatura en computación específicamente. Ubicamos el contexto principal de estudio en la situación de Costa Rica; no obstante, se incluyen componentes de literatura de alcance mundial, para enmarcar los resultados de la investigación.

El método de investigación utilizado fue una revisión bibliográfica sistemática. Los elementos más relevantes del método son desarrollados en la siguiente sección del artículo. Luego de la metodología, se presentan los principales elementos del contexto mundial sobre la licenciatura en computación y áreas afines. Aquí se hace un breve análisis de la evolución del grado de "licenciatura", se contextualiza el proceso de Bolonia, y se desarrolla lo relativo a la licenciatura en computación como grado académico. La siguiente sección de relevancia enmarca el grado de licenciatura en computación en el contexto de Costa Rica, en donde se hace, principalmente, un análisis de elementos de tipo de normativo, con el fin de explorar el estado de la situación que tiene dicho grado en licenciatura en computación en el caso de Costa Rica, en este momento. Los principales aspectos de análisis se desarrollan junto con la revisión bibliográfica en sí, el artículo termina con las conclusiones respectivas.

\section{Método}

Hart (1998) indica que una revisión bibliográfica usa las ideas contenidas en los textos para justificar un enfoque particular sobre un tema específico, la selección de métodos utilizados en el desarrollo de la revisión y la demostración de que dicha revisión aporta algo nuevo. Al respecto, Webster y Watson (2002) exponen que una revisión bibliográfica crea una base sólida para el avance del conocimiento, facilita el desarrollo de la teoría, cierra las áreas donde existe una gran cantidad de investigación, y descubre las áreas donde se necesita más investigación. Según Levy y Ellis (2006), como método de investigación, una revisión bibliográfica debe incluir características específicas de forma (metódica y con calidad), proporcionar una base sólida para 
doi: http://dx.doi.org/10.15359/ree.21-3.4

URL: http://www.una.ac.cr/educare

CORREO: educare@una.cr

la selección de una metodología de investigación y también demostrar que la investigación propuesta aporta algo nuevo al cuerpo general de conocimiento.

Mediante la taxonomía de clasificación para revisiones bibliográficas de Cooper (1988), se ha establecido para esta revisión un enfoque basado en prácticas o aplicaciones, un objetivo de identificación de ideas centrales, una cobertura representativa, una organización históricaconceptual y una audiencia de tomadores de políticas.

La búsqueda de información fue hecha en idioma inglés y español. Para identificar los elementos bibliográficos que serían incluidos en este trabajo, se realizaron búsquedas en bases de datos relevantes como EBSCO, ACM, Scopus, Springer, ScienceDirect, JSTOR, Academic Search Complete, Emerald, ERIC y Web of Science. Complementariamente, se realizaron búsquedas en Google Scholar. Algunos elementos bibliográficos especiales de interés para esta investigación no suelen estar incorporados en bases de datos bibliográficas. Los ejemplos más puntuales para esta situación son las normas, reglamentos, dictámenes, leyes, etc., que brindan elementos interesantes a esta investigación. Estos elementos fueron ubicados en un proceso de búsqueda minuciosa en diversas fuentes de referencia como sitios de internet de instituciones públicas de Costa Rica, entre otros. El contexto de la investigación es limitado. Se trata del estado de la situación del grado de licenciatura en computación en Costa Rica. Después de un análisis detallado, se seleccionaron 29 fuentes bibliográficas.

\section{El grado de licenciatura en computación y áreas afines: El contexto mundial}

En este apartado se presenta la situación del grado de licenciatura en computación y áreas afines en el contexto mundial.

\section{Breve análisis de la evolución del grado de “licenciatura” en computación ${ }^{6}$ y áreas afines}

Según Rüegg (1992), la licenciatura, así como el bachillerato, maestría y doctorado, son grados académicos que fueron institucionalizados en la antigua Edad Media por las universidades europeas. No obstante, en el contexto mundial actual, la licenciatura en computación o afines, que para efectos de simplificar la narrativa llamaremos en adelante "licenciaturas en computación" o "LC", como grado académico, no existe o tiene un alcance muy limitado. En Estados Unidos de Norteamérica y Canadá, existe el bachillerato como grado académico universitario inicial (Lunt et al., 2008; Topi et al., 2010; IEEE, 2014). En los países de la Unión Europea (UE), como resultado de la Declaración de Bolonia, se ha adoptado un sistema de grados universitarios de dos ciclos: (pre-grados y pos-grados). En este contexto, independiente de la disciplina de estudio,

${ }^{6}$ Referente temático asociado al estudio de pertinencia del grado de licenciatura abordado como ejemplo en este artículo.

4

Fulvio Lizano-Madriz y Carmen Cordero-Esquivel

Los artículos de la Revista Electrónica Educare del Centro de Investigación y Docencia en Educación de la Universidad Nacional, Costa Rica, se comparten bajo términos de la Licencia Creative Commons: Reconocimiento, No Comercial, Sin Obra Derivada 3.0 Costa Rica. Las autorizaciones adicionales a las aquí delimitadas se pueden obtener en el correo: educare@una.cr 
el bachillerato es considerado en Europa como el primer grado académico a obtener en una universidad. El grado de licenciatura como tal, al igual que en Estados Unidos de Norteamérica y Canadá, no existe (EHEA 1999). Esta situación también se presenta con un ligero matiz en el caso de Latinoamérica. Por su parte Contreras (2013) expone como un sub-producto específico del Proyecto Tuning América Latina, los resultados de una cuantificación reciente no exhaustiva de carreras relacionadas como computación, identificó en América Latina solo 7 "grados de licenciatura" relacionadas con la computación y 26"ingenierías" relacionadas con la computación.

En el contexto del proyecto de investigación que generó este documento, con los recursos disponibles, no es posible establecer de forma contundente por medio de la bibliografía, si la limitada presencia del grado de LC presente en América Latina, es el resultado de un proceso que está en evolución. No obstante, algunos fenómenos mundiales de referencia como el proceso de Bolonia (PB), pareciera que están forzando el debate hacia un sistema de grados simples como el establecido, según Kelo (2008) en la misma Declaración de Bolonia. De hecho, algunos como Brunner $(2008,2009)$ piensan que dicha declaración puede inspirar algunos procesos de adopción de algunos de sus principios en América Latina. Sin embargo, si existiera dicho proceso de adopción de un esquema más simple de pre-grados universitarios como el propuesto por la Declaración de Bolonia, se podrían, según Pritchard (2006), presentar procesos de resistencia al cambio y poca proactividad de las universidades, siendo a veces necesaria la implementación de dichos cambios por medio de procesos formales de generación de nueva legislación similar a lo ocurrido en el caso de Alemania.

En el caso de la LC, podría ser que estemos ante un panorama que imponga un cuestionamiento y replanteamiento de su existencia, con el fin de permitir esquemas más simples de grados universitarios. Hoy por hoy, se podría aceptar que la demanda de mano de obra calificada por parte de la industria, comercio, gobierno, etc., genera una absorción de mucho del recurso humano que se produce en las universidades. En este sentido, la academia enfrenta importantes retos para poder optimizar sus recursos, partiendo por el recurso humano esencial, para procesos de docencia e investigación. En este punto, Roberts (2000) considera que ha identificado que la escasez de mano de obra calificada tiene un impacto directo en la academia, al "mover" a los buenos y a las buenas profesionales fuera de esta para suplir necesidades de la industria, comercio, gobierno, etc.

No obstante, según Soto (2006), este cuestionamiento y eventual replanteamiento hacia un sistema degradosmássimplea nivel de pre-grados (consoloungrado debachillerato), ineludiblemente implicará considerar muchos aspectos tales como modernidad, formación y desarrollo tecnológico, calidad del trabajo en redes, financiación y un nuevo modelo universitario en general.

Sea cual sea el caso, el proceso de Bolonia se impone como el modelo de referencia, más reciente, actual y estudiado en esta materia. 
doi: http://dx.doi.org/10.15359/ree.21-3.4

URL: http://www.una.ac.cr/educare

CORREO: educare@una.cr

\section{El proceso de Bolonia (PB)}

El PB surge, según la Declaration (EHEA,1999) y Fejes (2006), para implantar en la Unión Europea (UE) un proceso de normalización de ideas, movilidad de estudiantes y docentes y, finalmente, la implementación de un esquema de gestión académica universitaria que sea transparente. No obstante, según Fejes (2006), al ser un proceso de adopción voluntaria, la dinámica de la armonización de los procesos académicos entre gran cantidad de miembros de la $\mathrm{UE}$, ha provocado que el proceso sea visto por muchas universidades como un proceso inevitable.

EI PB establece principios para muchos aspectos relacionados con la dinámica académica. Para los efectos que interesa en este documento, de acuerdo con Hawes (2005), el PB propone la adopción de un sistema simple de grados basado en dos ciclos principales: pregrado y posgrado. El acceso al segundo ciclo requiere la finalización exitosa de los estudios del primer ciclo, el cual tendrá una duración mínina de tres años. Este único grado ofrecido en el primer ciclo es comúnmente conocido como "bachillerato" y se considerará relevante para el mercado laboral europeo como representativo de un nivel adecuado de formación profesional. Según la Declaration (EHEA, 1999), el segundo ciclo tiene dos opciones: maestría o doctorado.

Como indica Fejes (2006), se espera que el grado de maestría se obtenga en 2 años y el de doctorado en 3-4 años. En general, Fejes (2006) sostiene que el PB es un proceso neoliberal que se basa en el constructivismo y autorregulación de forma tal que el "gobierno" descansa en las mismas unidades académicas (universidades). Agrega Fejes (2006) que los procesos de estandarización y movilidad se implementan de diversas formas y el más evidente es el establecimiento de un sistema único de créditos Ilamado "Sistema de Transferencia de Créditos Europeos" (ECTS - European Credit Tranfer System). Mientras que Karseth (2006) expone que estos principios (sistema simple de grados, ECTS, etc.), buscan la restructuración de los planes de estudio para ir hacia un sistema unificado que facilite la movilidad entre las diferentes regiones europeas. En este sentido, el PB se convierte en un referente "interesante" a la hora de pensar en la restructuración de planes de estudios.

En la bibliografía se ha documentado como dato general una cantidad importante de información referente a las ventajas y desventajas del PB. Las principales ventajas y desventajas del PB sin ninguna catalogación en especial, se muestran en la Tabla 1.

Hablando de ejemplos concretos de implementación del PB, encontramos en nuestro contexto con el proyecto Tuning que, según Aboites (2010), es derivado directamente del PB y pretende impulsar un modelo basado en competencias en América Latina. Asimismo, Contreras (2013) expone que el proyecto Tuning considera, aparte de otras áreas de conocimiento, las carreras de computación y afines. Por otro lado, Klimesš y Habiballa (2006) presentaron una experiencia real de integración del PB en planes de estudio relacionados con ciencias de la computación en el Departamento de Ciencias de la Computación de la Universidad de Ostrava, República Checa. Esta implementación incluía los tres niveles de grados sugeridos por el PB (bachillerato, maestría y doctorado). 
Tabla 1: Ventajas y desventajas del PB

\begin{tabular}{l}
\hline Ventajas \\
\hline En un contexto especifico (Italia), se encontró que \\
entre el staff académico existe una percepción gene- \\
ralizada de que el PB permite un mayor intercambio \\
de estudiantes, la reorganización de los programas \\
de estudio de acuerdo con la nueva estructura de los \\
estudios y, finalmente, la búsqueda de socios para la \\
enseñanza de la cooperación y la colaboración en la \\
investigación (Aittola et al., 2009).
\end{tabular}

EL PB permitió, en lugares como España, replantear teóricamente la formación universitaria para hacerla más acorde con la realidad laboral (González-Serrano, 2011).

El PB impulsa un proceso de comparabilidad creciente de grados y una consecuente transparencia de los logros de los graduados. Este proceso obliga a las universidades a generar procesos innovadores de gestión que les permitan competir por atraer nuevo estudiantado (Wiepcke, 2009).
Desventajas

En contextos como el latinoamericano, el PB enfrentaría una evidente falta de espacio común, el legado colonial, anacrónicas políticas económicas y sistemas de enseñanza y, finalmente, ciertas tendencias modernas y limitantes comunes tales como proliferación de instituciones de enseñanza terciarias (universidades, institutos preuniversitarios, universidades privadas, etc.) fortalecimiento de universidades no estatales (47\%), diversidad, reconocimiento a esquemas tradicionales, bloqueo de nuevas iniciativas de formación, débil investigación, etc.) (Brunner, 2008).

En aquellos países donde existía un proceso de pregrados que duraba 4-5 años, con bachillerato o licenciatura, se produjeron mayores críticas al "reducir" el pregrado a un solo diploma (bachillerato) de 3 años (Cardoso, Portela, Sá y Alexandre, 2008).

En contextos como el latinoamericano, el PB tendría como obstáculos el bajo financiamiento estatal, estructuras rígidas y desaliento a la internacionalización (Brunner, 2009).

Los objetivos del PB son muy ambiciosos (GonzálezSerrano, 2011).
Existe un impacto positivo del PB al inspirar la colaboración entre universidades en contextos como Portugal-España y Latinoamérica (Brunner, 2009).

Nota: Elaboración propia.

\section{La LC como grado académico}

Tal como se mencionó anteriormente y como lo indica Contreras (2013), la LC como grado académico está presente de forma marginal en Latinoamérica.

De acuerdo con Declaration (EHEA, 1999), en acatamiento de las consideraciones del PB en la UE, a nivel de pregrado solo existe una formación mínima de 3 años que confiere el diploma de bachillerato. Los otros 2 grados posibles a nivel universitario son la maestría y doctorado con una duración usual de 2 y 3-4 años respectivamente. Al respecto, Kelo (2008) expone este modelo de grados (bachillerato-maestría-doctorado), que no incluye el grado de licenciatura, es ampliamente utilizado en gran parte del mundo.

Por su parte, IEEE (2014) indica que, en el contexto de USA-Canadá, existe el nivel de grado el bachillerato. Un ejemplo emblemático de un marco de referencia para este esquema fue 
doi: http://dx.doi.org/10.15359/ree.21-3.4

URL: http://www.una.ac.cr/educare

CORREO: educare@una.cr

propuesto desde el 2005. Entre las disciplinas consideradas en esta propuesta están la ingeniería en computación, ciencias de la computación, sistemas de información, tecnología de información e ingeniería de software. En este mismo contexto, la IEEE (2014) menciona que se han propuesto modelos o recomendaciones de planes de estudios específicos, para bachillerato, en CIENCIAS DE LA COMPUTACIÓN, mientras que Topi et al. (2010) exponen que en sistemas de información también. Asimismo, el IEEE (2014) indica que en ingeniería de software sucede lo mismo y en tecnología de información (IEEE , 2014; Lunt et al., 2008). Si bien es cierto, estas propuestas curriculares no son de acatamiento obligatorio, constituyen un referente importanteen el contexto del presente documento.

\section{La LC en el contexto costarricense}

En este apartado se presenta la situación del grado de licenciatura en computación y áreas afines en el contexto costarricense.

En Costa Rica, el Consejo Nacional de Rectores (CONARE), específicamente la Oficina de Planificación de la Educación Superior, estableció en el 2004 un convenio sobre la nomenclatura de grados y títulos de la educación superior universitaria estatal. Este convenio estableció que existen 3 niveles de caracterización de los grados universitarios estatales. El primer nivel se conoce como "pregrado" y contempla el diplomado y el profesorado. El segundo nivel llamado "grado" incluye el bachillerato universitario y la licenciatura. Finalmente, el tercer nivel llamado "posgrado" considera tres alternativas: especialidad profesional, maestría y doctorado académico (Universidad de Costa Rica, Consejo Universitario, 2004). La Figura 1 ilustra el estado de los grados académicos en Costa Rica y los compara con la normalidad de otros contextos externos.

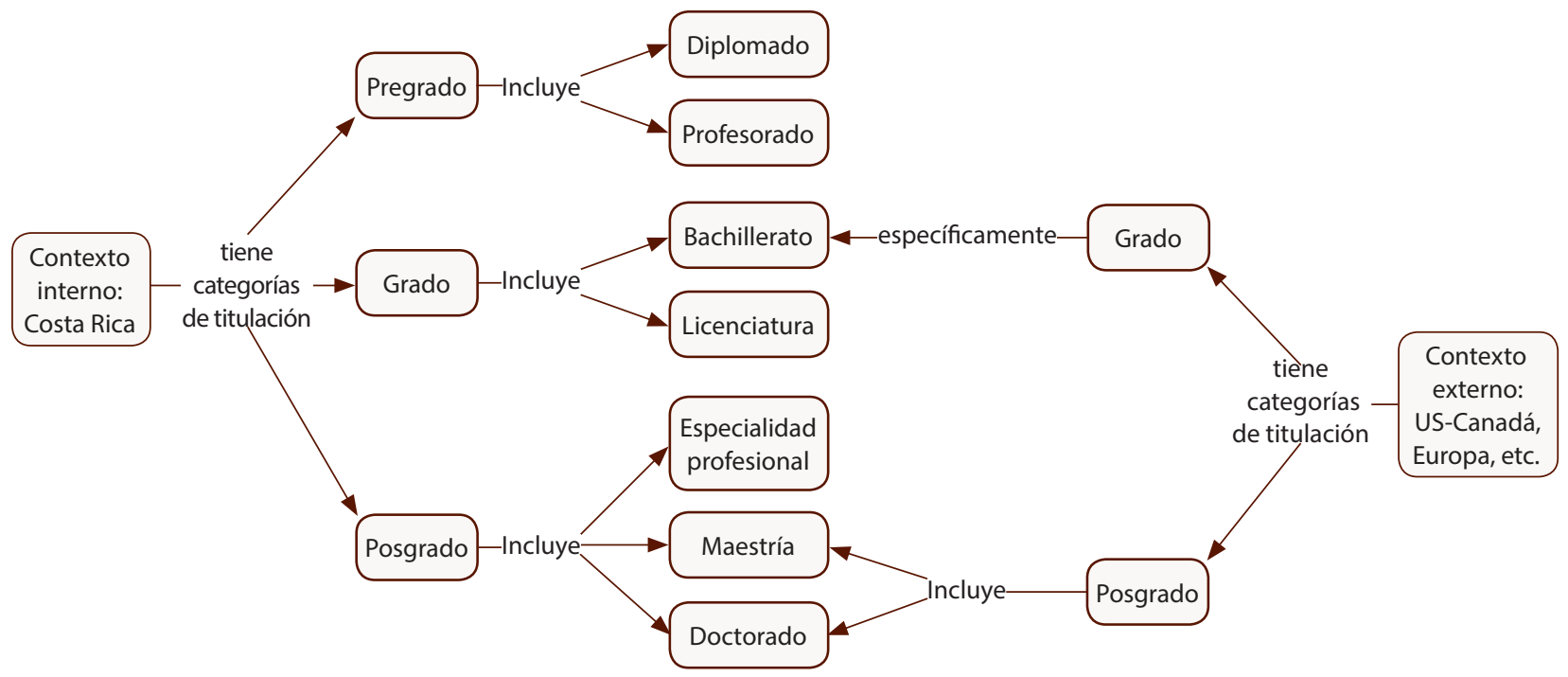

Figura 1: Grados académicos en Costa Rica y otros contextos externos.

Nota: Elaboración propia. 
De lo anterior se desprende que todas las LC impartidas en las universidades públicas en Costa Rica obedecen a la denominación de "grado" universitario. Dentro de este contexto, todas las LC que se imparten en las universidades privadas en Costa Rica tienen la misma validez legal que las impartidas en las universidades públicas estatales, Ley N. ${ }^{\circ} 6693$ (Asamblea Legislativa de la República de Costa Rica, 1981).

En el ámbito que interesa explorar en este documento, el grado de LC pareciera que, por tradición, tiene cierta preferencia sobre los grados de maestrías. Esto, para aquellos casos de profesionales en computación, con un grado de bachillerato, que piensan en optar por empleos a nivel gubernamental. De acuerdo con Siu (2014), el grado de bachillerato es el mínimo aceptado por los grandes empleadores del sector público del gobierno en Costa Rica. Al explorar las opciones de continuar estudios profesionales después del grado de bachillerato, los grupos graduados en computación en Costa Rica tienen diversas opciones a nivel nacional, tal como se mencionó antes. Sin embargo, expone Vessuri (2008), aparte del alto costo de los posgrados que existen en Latinoamérica, la preferencia por el grado de LC podría haberse originado en cierta percepción de que, el grado de LC tiene la misma validez práctica que una maestría, esto, al menos, desde el punto de vista de reconocimiento económico.

El reconocimiento económico de los grados académicos en la mayor parte del sector público de Costa Rica se implementa por medio del llamado régimen de Carrera Profesional. En el artículo 1 de la Resolución DG-064-2008, la Dirección General del Servicio Civil define el citado régimen como:

Denomínese Carrera Profesional al incentivo económico por medio del cual se reconoce el mérito del funcionario profesional, que presta sus servicios en las instituciones del Poder Ejecutivo, que cumpla con los requisitos señalados en el Artículo $3^{\circ}$ de este cuerpo normativo y satisfaga alguno de los siguientes factores:

a) Grados y posgrados académicos. (Dirección General de Servicio Civil, República de Costa Rica, 2008, p. 3)

La percepción sobre la similar validez del grado de licenciatura y el de las maestrías podría haber tenido origen en lo normado en Resolución DG-080-96 de la Dirección General del Servicio Civil (Dirección General de Servicio Civil, República de Costa Rica, 1996), la que establecía en su artículo 5, inciso "a", una serie de ponderaciones para los diferentes grados académicos. De esta forma, para el grado de licenciatura se estableció un "puntaje" de 16 y para el de maestría de 32. No obstante, al final del inciso "a", se indica:"En el caso de que la maestría haya sido obtenida con el grado bachillerato, el puntaje será únicamente de 16 puntos" (Dirección General de Servicio Civil, República de Costa Rica, 1996, p. 5). 
doi: http://dx.doi.org/10.15359/ree.21-3.4

URL: http://www.una.ac.cr/educare

CORREO: educare@una.cr

Esta evidente "desventaja" de optar por un grado de maestría con base en el grado de bachillerato quedó eliminada con la misma resolución DG-064-2008, que sustituyó a la DG080-96. Ya en la DG-064-2008 se establece, en el artículo 4, que el grado de licenciatura tendrá un puntaje de 16 (6 puntos adicionales a los del grado de bachillerato), el grado de maestría de 32 (22 puntos adicionales a los del grado de bachillerato) (Dirección General de Servicio Civil, República de Costa Rica, 2008). En este sentido, se individualiza cada uno de los grados de licenciatura y de maestría estableciéndose puntajes diferentes, adicionales al grado de bachillerato, para cada uno de estos.

Esta resolución DG-064-2008 (Dirección General de Servicio Civil, República de Costa Rica, 2008), de aplicación generalizada en todas aquellas instituciones dependientes de la Autoridad Presupuestaria de Costa Rica, establece tácitamente una diferente "jerarquía" para los grados de licenciatura y de maestría. En abono a esto, para los efectos de la discusión elaborada hasta el momento, el grado de licenciatura se convierte de hecho en "opcional" e innecesario en aquellos casos en donde se cuenta con un grado de bachillerato y de maestría al mismo tiempo. Por su parte, en la Resolución DG-120-2005 de la Dirección General del Servicio Civil, se indica en su artículo 2:

Las maestrías o doctorados declarados atinentes en el correspondiente Manual, serán aceptados para aquellas clases que requieren el grado de Bachillerato o Licenciatura, [subrayado añadido] siempre y cuando el candidato, en la eventualidad de un nombramiento o ascenso, este debidamente incorporado al colegio profesional respectivo. (Dirección General de Servicio Civil, República de costa Rica, 2005 p. 2)

En resumen, en el caso de profesionales en computación con un grado de bachillerato que piensan optar por puestos a nivel gubernamental, resulta más ventajoso, desde el punto de vista de ingresos, optar por un grado de maestría luego del de bachillerato. Toda la normativa anteriormente citada es útil para establecer lo anterior en el sector público. No se consideró analizar normativa del sector privado, debido a que no existe obligatoriedad ni uniformidad en esta misma. El análisis hecho aquí pretende establecer la "importancia relativa económica" del grado de licenciatura, y por supuesto de la LC, en un contexto específico para Costa Rica (el sector público).

\section{Conclusiones}

El artículo es un extracto de un documento que contiene un diagnóstico de la situación actual de las licenciaturas ofrecidas por la Escuela de Informática de la Universidad Nacional de Costa Rica. Lo presentado en este artículo corresponde a la revisión bibliográfica hecha para el análisis del estado del grado de las LC en el contexto costarricense. Algunos elementos de alcance externo a Costa Rica fueron incluidos con el objetivo de brindar un marco de referencia inicial. 
La característica fundamental del grado de LC es su inexistencia. En casi todo el mundo, las LC, como grado académico, no existen o tienden a ser suprimidas. Esto último como resultado de la generalización de modelos de organización académica, como el conocido proceso de Bolonia (PB), del cual se han resumido sus principales ventajas y desventajas en la Tabla 1. Si bien es cierto que a nivel latinoamericano existen todavía algunos grados de licenciatura, estos son relativamente pocos, pues se evidencia una fuerte presión a discutir y adoptar modelos alternos como el Tuning, el cual pretende tropicalizar modelos como el mismo PB. En la bibliografía se reportan ejemplos exitosos de estos procesos de migración, principalmente en Europa.

En el contexto nacional, el grado de LC ha dejado de tener el atractivo, debido a la similitud con el grado de maestría, en cuanto al reconocimiento económico laboral a nivel de gobierno central. En este documento se demuestra que en el caso de profesionales en computación, con un grado de bachillerato que piensan optar por puestos a nivel gubernamental, resulta más ventajoso obtener un grado de maestría luego del de bachillerato. La histórica "importancia relativa económica" de los grados de licenciatura, incluyendo las LC, no es más que eso: historia.

\section{Referencias}

Aboites, H. (2010). La educación superior latinoamericana y el proceso de Bolonia: De la comercialización a la adopción del proyecto Tuning de competencias. Educación Superior y Sociedad, 15(1), 25-44. Recuperado de http://ess.iesalc.unesco.org.ve/index.php/ess/ article/view/367/306

Aittola, H., Kiviniemi, U., Honkimäki, S., Muhonen, R., Huusko, M. y Ursin, J. (2009). The Bologna process and internationalization - consequences for Italian academic life. Higher Education in Europe, volume, 34(3-4), 303-312. doi: https://doi.org/10.1080/03797720903355521

Soto, D. (2006). La universidad latinoamericana en el siglo XXI. Revista Historia de la Educación Latinoamericana, 8, 113-136. Recuprdo de http://revistas.uptc.edu.co/index.php/historia educacion latinamerican/article/view/3598/3194

Asamblea Legislativa de la República de Costa Rica. (21 de diciembre de 1981). Ley 6693. Crea Consejo Nacional Enseñanza Superior Universitaria Privada, CONESUP. La Gaceta, Diario Oficial de la República de Costa Rica, Año 103, N. ${ }^{\circ} 243$.

Brunner, J.J. (2008). El proceso de Bolonia en el horizonte latinoamericano: Límites y posibilidades. Revista de Educación, número extraordinario, 119-145. Recuperado de http://www. revistaeducacion.mec.es/re2008/re2008 06.pdf

Brunner, J. J. (2009). The Bologna process from a Latin American perspective. Journal of Studies in International Education, 13(4), 417-438. doi: https://doi.org/10.1177/1028315308329805 
doi: http://dx.doi.org/10.15359/ree.21-3.4

URL: http://www.una.ac.cr/educare

CORREO: educare@una.cr

Cardoso, A. R., Portela, M., Sá, C. y Alexandre, F. (2008). Demand for higher education programs: The impact of the Bologna process. CESifo Economic Studies, 54(2), 229-247. doi: https:// doi.org/10.1093/cesifo/ifn013

Universidad de Costa Rica, Consejo Universitario. (2 de abril, 2004). Sesión 4866. Política académica. Convenio de nomenclatura de grados y títulos de la educación superior universitaria del martes 9 de marzo, 2004. La Gaceta Universitaria. Órgano oficial de comunicación de la Universidad de Costa Rica, 28(7), p. 4. Recupereado de http://www. cu.ucr.ac.cr/uploads/tx ucruniversitycouncildatabases/officialgazette/2004/g07-2004. pdf\#page $=14$

Contreras, J. L. (Ed.). (2013). Tuning America Latina. Educación Superior en America Latina: reflexiones y perpectivas en Informática. Bilbao: Universidad de Deusto. Recuperado de http://tuningacademy.org/wp-content/uploads/2014/02/Reflnformatics LA SP.pdf

Cooper, H. M. (1988). Organizing knowledge synthesis: A taxonomy of literature reviews. Knowledge in Society, 1(1), 104-126. doi: https://doi.org/10.1007/BF03177550

European Higher Education Area (EHEA). (1999). The Bologna declaration of 19 june 1999. Joint declaration of the European Ministers of Education.

Dirección General de Servicio Civil, República de Costa Rica. (28 de febrero, 2008). Resolución N. ${ }^{\circ}$ DG-064-2008. Recuperado de http://www.mag.go.cr/acerca del mag/circulares/rec hum-resolucion-064-2008-carr-prof.pdf

Dirección General de Servicio Civil, República de Costa Rica. (3 de octubre, 1996). Resolución N. ${ }^{\circ}$ DG-080-1996. Recuperado de http://www.mag.go.cr/acerca del mag/circulares/rec hum-resolucion-064-2008-carr-prof.pdf

Dirección General de Servicio Civil, República de Costa Rica. (19 de abril de 2005). Resolución N. ${ }^{\circ}$ DG-120-2005.

Fejes, A. (2006). The Bologna process - governing higher education in Europe through standardization. Revista española de educación comparada, 12, 203-232. Recuperado de http://www.sc.ehu.es/sfwseec/reec/reec12/reec1207.pdf

González-Serrano, M. D. C. C. (2011). Una aproximación a los aspectos positivos y negativos derivados de la puesta en marcha del Plan Bolonia en la Universidad Española. REJIE: Revista Jurídica de Investigación e Innovación Educativa, 4, 91-104. Recuperado de https:// dialnet.unirioja.es/servlet/articulo?codigo $=4584986$

Hart, C. (1998). Doing a literature review: Releasing the social science research imagination. London: Sage Publications. 
Hawes, G. (Junio, 2005). Las claves del Programa de Bolonia en perspectiva de la universidad latinoamericana y del Caribe. Presentado en la Reunión de la Comisión de Educación, Cultura, Ciencia, Tecnología y Comunicación del Parlamento Latinoamericano. Reunión llevada a cabo en Universidad de Talca, Santiago de Chile. Recuperado de https://www. academia.edu/1177087/Las claves del Programa de Bolonia en perspectiva de la universidad latinoamericana y del Caribe

Institute of Electrical and Electronics Engineers, IEEE. (2014). Software Engineering 2014. Curriculum Guidelines for Undergraduate Degree Programs in Software Engineering. IEEE Computer Society y Association for Computing Machinery.

Karseth, B. (2006). Curriculum restructuring in Higher Education after the Bologna Process: A new pedagogic regime? Revista española de educación comparada, 12, 255-284. Recuperado de http://www.sc.ehu.es/sfwseec/reec/reec12/reec1209.pdf

Kelo, M. (Ed.). (2008). Beyond 2010. Priorities and challenges for higher education in the next decade. Bonn: Lemmens. Recuperado de http://www.aca-secretariat.be/fileadmin/aca docs/images/members/2008 Beyond 2010 - Priorities and challenges for higher education in the next decade.pdf

Klimesš, C. y Habiballa, H. (2006). Flexible computer science university studies. ACM SIGCSE Bulletin, 38(3), 338-338. doi: https://doi.org/10.1145/1140123.1140246

Levy, Y., \& Ellis, T. J. (2006). A systems approach to conduct an effective literature review in support of information systems research. Informing Science: International Journal of an Emerging Transdiscipline, 9, 181-212. Recuperado de http://www.inform.nu/Articles/Vol9/ V9p181-212Levy99.pdf

Lizano, F. y Cordero, C. (2015). Estudio de factibilidad y propuesta de creación de nuevo(s) programas a nivel de posgrado en computación para la Escuela de Informática (0001-14). Heredia: Escuela de Informática, Universidad Nacional.

Lunt, B. M, Ekstrom, J. J., Gorka, S., Hislop, G., Kamali, R., Lawson, E., ... Reichgelt, H. (2008). Curriculum Guidelines for Undergraduate Degree Programs in Information Technology. IEEE Computer Society y ACM Association for Computing Machinery.

Pritchard, R. (2006). Trends in the restructuring of German universities. Comparative Education Review, 50(1), 90-112. doi: https://doi.org/10.1086/498330

Roberts, E. (2000). Computing education and the information technology workforce. ACM SIGCSE Bulletin, 32(2), 83-90. doi: https://doi.org/10.1145/355354.355383 
doi: http://dx.doi.org/10.15359/ree.21-3.4

URL: http://www.una.ac.cr/educare

CORREO: educare@una.cr

Rüegg, W. (Ed.). (1992). A History of the University in Europe (Vol. 1: Universities in the Middle Ages). Cambridge: University Press.

Siu, M. (17 de noviembre de 2014). Sector público excluye técnicos sin bachillerato. La República. Recuperado de https://www.larepublica.net/noticia/sector publico excluye tecnicos sin bachillerato /

Topi, H., Valacich, J. S., Wright, R. T., Kaiser, K., Nunamaker, J. F. Jr., Sipior, J. C., \& de Vreede, G. J. (2010). IS 2010: Curriculum guidelines for undergraduate degree programs in information systems. Communications of the Association for Information Systems, 26(1), 359-428.

Vessuri, H. (2008). Competición y colaboración en un contexto de multiplicación de "Centros de atracción" y "Desiertos yermos". Revista de la Educación Superior, 37(148), 123-139. Recuperado de http://publicaciones.anuies.mx/pdfs/revista/Revista148 S2A5ES.pdf

Webster, J., \& Watson, R. T. (2002). Analyzing the past to prepare for the future: Writing a literature review. MIS Quarterly, 26(2), 13-23.

Wiepcke, C. (2009). Employability in the Bologna process: An area of tension between society, businesses and students. The International Journal of Learning, 16(4). 435-446. doi: https:// doi.org/10.18848/1447-9494/CGP/v16i04/46237 